\title{
Web 2.0: Beyond the Concept \\ Practical Ways to Implement RSS, Podcasts, and Wikis
}

\author{
By Karen Huffman, National Geographic Society
}

\begin{abstract}
This article focuses on how the National Geographic Society's Libraries \& Information Services has implemented RSS, podcasts and wikis with suggested practical application for academic libraries. The article is an adaptation from a presentation given during the Special Libraries Association's Annual Conference, June 2006 (Huffman \& Ferry, 2006).
\end{abstract}

\section{The Sparks That Ignited Us....}

National Geographic's Libraries \& Information Services (LIS) discovery and implementation of technologies dubbed "Web 2.0" has been an evolutionary, rather than revolutionary process. We watched the trends to evolve our products and services. In 2005 we began seeing online news sources like BBCnews.com offering alternative ways to access their content. New RSS-labeled icons appeared in the margins of websites with links to directories of feeds. These RSS directories generally organized their feeds either topically or by news section and often included frequently asked questions about RSS as well as recommended feed readers. As information organizers (and multi-taskers who streamline their work processes wherever possible), we initially saw RSS feeds as a way to keep current with news sources, blogs, and research sites that were regularly publishing content through XML-based feeds!

We saw the arrival of podcasts on the heels of RSS. In January and February, 2005, the Pew Internet and American Life Project conducted a survey, and found 22 million, or $11 \%$, Americans age 18 or older owned an iPod or MP3 player. Apple's freely-available iTunes library brought podcasting to the mainstream with the launch of its "Podcasts" directory in June 2005. iTunes' integrated approach offered the ability to easily locate podcasts, subscribe to RSS feeds, and transfer them to an iPod. "In the first two days of availability...iTunes customers subscribed to more than 1 million podcasts" (Laue, 2005). Within the same month, companies like ABC news, Disney Online, and ESPN.com launched large collections of podcasts via iTunes ("ABC News...," 2005; Pogue, 2005).

Colleges and universities were making the news. Duke University in Durham, North Carolina, was an early adopter of iPods. As an experiment in the fall of 2004, the University gave all incoming freshmen iPods to "help invigorate the university's academic life" (Read, 2005). Although the year-long study found that not all curriculums necessitated the use of iPods, the devices did prove beneficial for some and would continue to be supported by the courses that required the devices. Apple also saw an opportunity to support educational initiatives of colleges and universities by launching iTunes University (iTunes $U$ ), a freely-available digital archives and Web hosting solution (Gordon-Murnane, 2005; Read, 2005; Stephens, 2005; Udell, 2006).

Wikipedia's exponential growth since its inception in mid-January of 2001 has intrigued and confounded the professional community, bringing attention to this digital, collaborative encyclopedia among writers and analytics in the news, professional journals, magazines, and blogs and as "hot topics" during conferences. In March, 2006, Wikipedia reached one million articles, and as of July, 2006, it now contains more than 1.28 million articles (Schiff, 2006; Wikipedia, 2006).

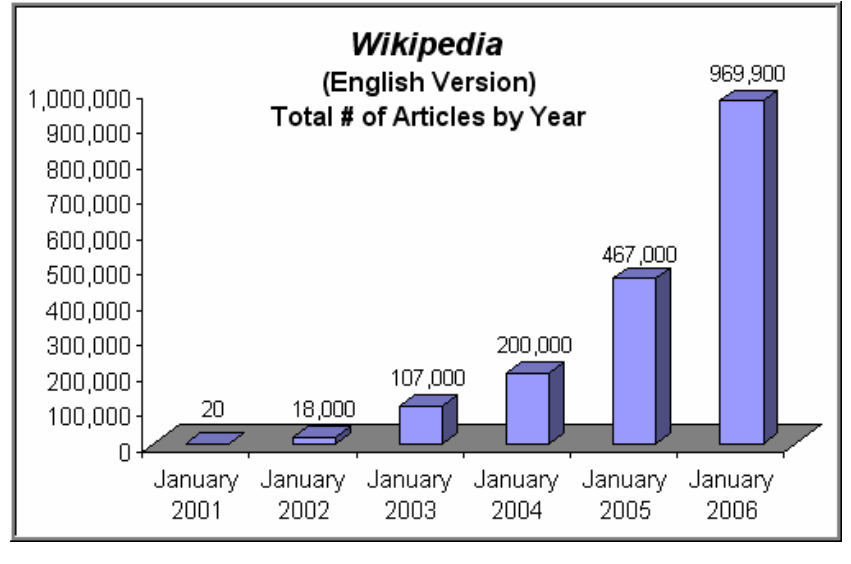


New wiki-based websites were appearing daily. Professional literature began defining wikis, discussed their historical evolution, compared wiki engines and hosted solutions, reviewed advantages and disadvantages of wikis, and offered examples of practical implementation efforts. As a result, National Geographic's Libraries started envisioning possible benefits for using collaborative applications such as wikis (Chawner, 2006; Clyde, 2005; Fichter, 2006; Ishizuka, 2005).

\section{National Geographic's Libraries Applied What We Learned}

We took a pragmatic approach with Web 2.0 applications through first-hand experience. As Greg Notess expressed in the May/June, 2006, issue of Online: "Rather than debate the overall merits of the 2.0 movement, information professionals should explore the territory, techniques, and examples to find the most useful applications in your own work environment." We realized that Web 2.0 was more about a concept on how the Web was evolving from flat HTML-based pages (i.e. Web 1.0) into interactive, social areas that integrated easily with people's lifestyles. We also knew that not everything we tried would work, but if we didn't explore and "play" with applications to understand how they worked, we could not see what potential they might have for our organization and its community.

We continue to offer products and services through traditional channels. For example, we still market through our outreach/liaison program, email newsletter, brochures, and flyers. Our Libraries website offers a rich collection of products and services including links to our catalogs, subject research guides, subscription databases, and training class descriptions. Staff can choose weekly training and learning opportunities from a variety of formats: hour-long classes, 20-minute coffee and cookie breaks, one-onone sessions, and online training tutorials and e-learning materials.

Web 2.0 applications offered the ability to evolve our content and services and develop new ways to market, present, and further expand our research offerings. It was not costly to experiment with different tools. We migrated our Libraries website to LunarPages.com, a Web hosting company that charges minimal yearly fees (about $\$ 100$ a year) and supports industry-standard Web server configuration. By moving our website to a new hoster, we had a "sandbox," or space, to start testing our ideas. Our largest success stories to date have included our implementation of RSS, podcasts, and wikis. We have also experimented with online communities and blogs but have had less internal traction for these applications.

RSS and Podcasts. About a year ago, we started building a directory of recommended RSS feeds for our customers. We also offered several 20-minute "coffee breaks" to introduce RSS and teach how to set up a Web-based reader like Bloglines.com to aggregate feeds for research purposes. With the launch of our new Libraries website in September, 2005, we ran streaming RSS feeds on our Libraries home page using an Open Source PHP parser called Magpie RSS. In contrast to our research-focused implementation efforts, two large local public library systems described how they had implemented RSS for marketing their services and events during last spring's annual Computer in Libraries Conference in Washington, D.C. 


\section{Directory of Recommended RSS Feeds for Our Researchers}

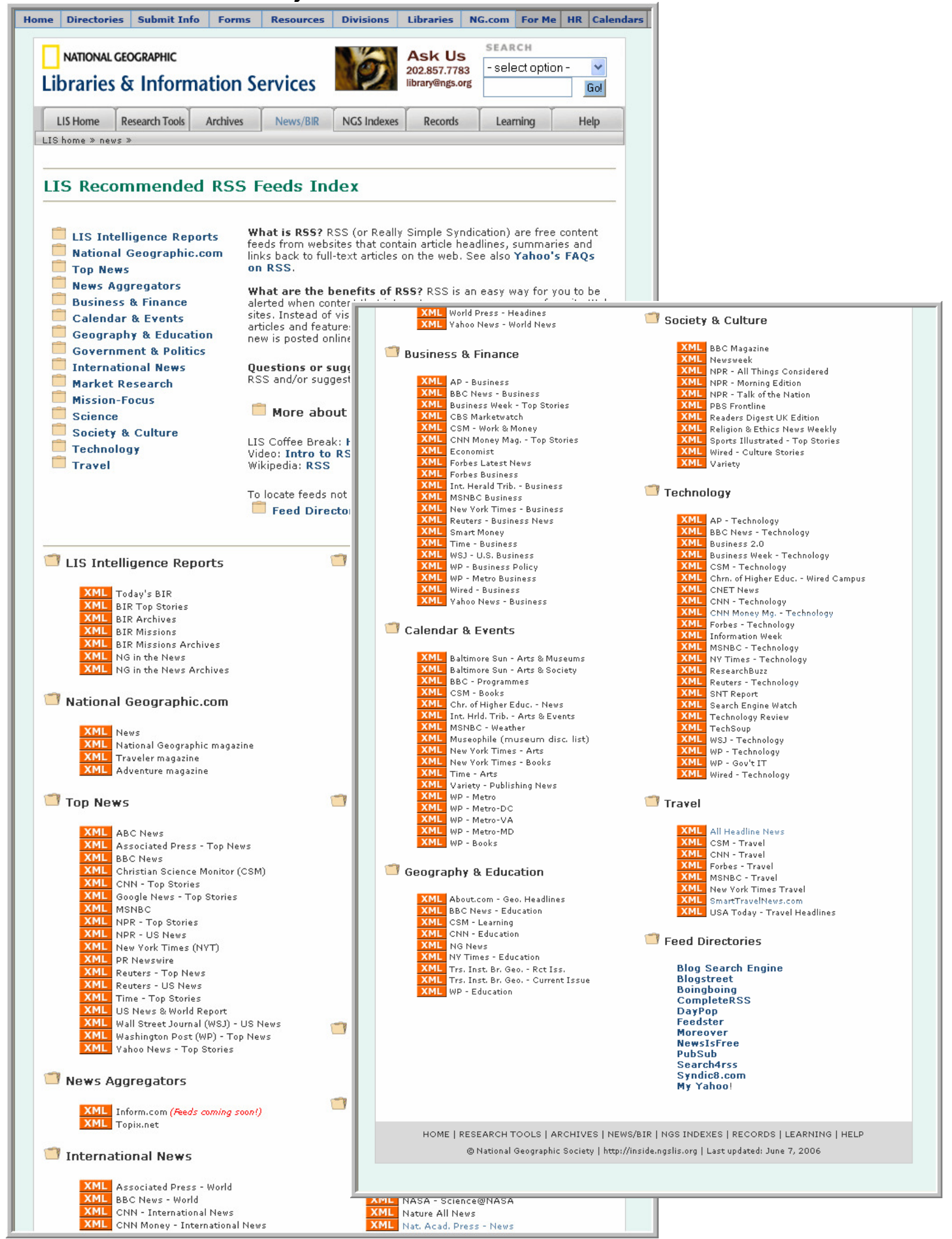


Library's Home Page: RSS feeds from BBC News and New York Times (right sidebar)

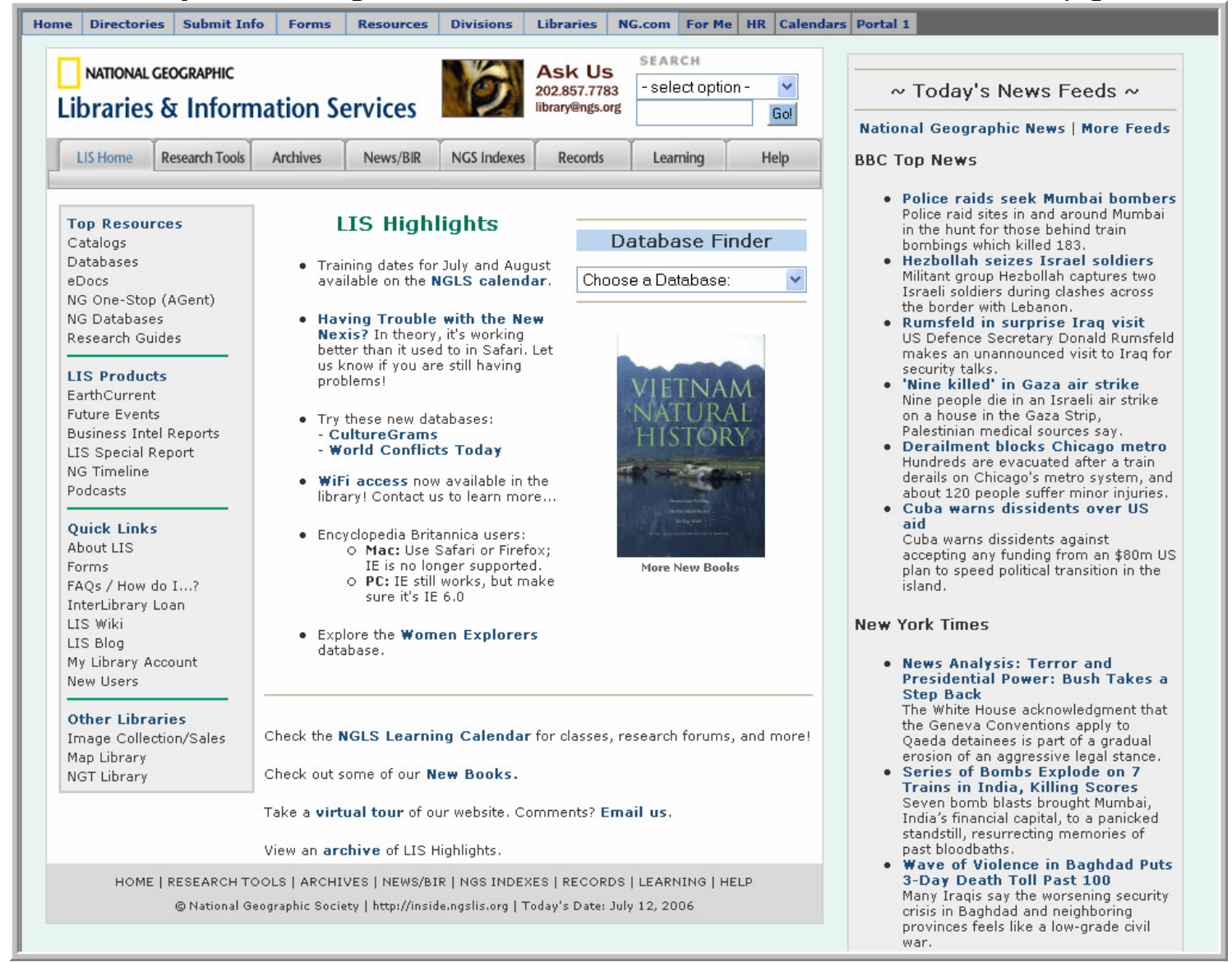

With some experience and understanding of RSS, we decided to explore podcasting. Our user community is from demographically diverse age groups, and we realized that not everyone wanted to receive information in the same way. Podcasting offered an additional marketing and information delivery channel to augment (not replace) our other marketing efforts.

National Geographic was fortunate to have the internal resources to do this: We have a studio and recording/editing equipment in our Audiovisual division; streaming audio capabilities on the desktop; available bandwidth (okayed by Information Systems \& Technology [IS\&T]); and our corporate intranet as a central means for distribution. We also are fortunate to have library staff with good radio voices and others with experience in radio writing and music production. We offered the opportunity to "try out" as broadcasters to all library staff - not just the report writers.

In October, 2005, we developed and branded a handful of podcasts from content we were already producing. These podcasts laid the groundwork for our weekly 20-minute podcasts based on our daily "Business Intelligence" and "National Geographic in the News" reports, our twice-weekly science-focused report called "EarthCurrent," our weekly "Missions Report," and monthly topically-focused "Special Reports." Our archivist started monthly recordings of an "NG History Moment," and shortly after we began to podcast the "Trends Analysis Group Quarterly Report."

Podcasting has helped us reach our younger staff at the Society and to look "cool" to the more technically-savvy staff. We also wanted to educate our executive staff on podcasting and its potential for the consumer market through practical applications and easy delivery channels. For an executive retreat, we uploaded NG content that was ready for podcasting onto new iPods. The executives "got it." NG 
began an enterprise podcast in August, spurred on by a library-led cross-divisional group that compiled assets, initiated enterprise branding, and negotiated with iTunes.

Wikis. As we were redesigning and building our new Libraries intranet site last summer and fall, we started experimenting with wikis. Wikis are online collaborative communities that lend themselves to continuous editing and refinement of content. They work best at aggregating and distilling shared knowledge and include the ability to track article evolution so that content often reflects a blend of voices.

As mentioned earlier, we followed the news about Wikipedia and reviewed other research-focused wikis that might offer potential resources for our editorial groups. Many wikis aggregate collections of knowledge and are good starting points for understanding a topic or finding links to additional resources. So, we began teaching our patrons about these exploding social applications, pointing them to potential research sources.

After formulating some initial ideas, we decided to implement a wiki. In August, 2005, we launched the "Libraries \& Information Services Wiki" (LIS Wiki) using MediaWiki, an Open Source application originally developed for Wikipedia. Our library staff began using the LIS Wiki by adding their resumes including pictures and anecdotal information about their interests, animals, and children. We decided to aggregate in the wiki our 20-minute coffee-break handouts, compile quick reference materials, and convert dictionary-like pages including our "Records and Archives Classification Dictionary" and our "NG Lingo" list for new employees. Within less than a year, our LIS Wiki has expanded to include intra- and interdivisional projects where team members can brainstorm and develop ideas for our consumer market, collaborate on status reports, and share research endeavors and information on trends, including links to working documents.

\section{Examples of LIS Wiki home page, News Collection project status, and "NG Lingo" dictionary}

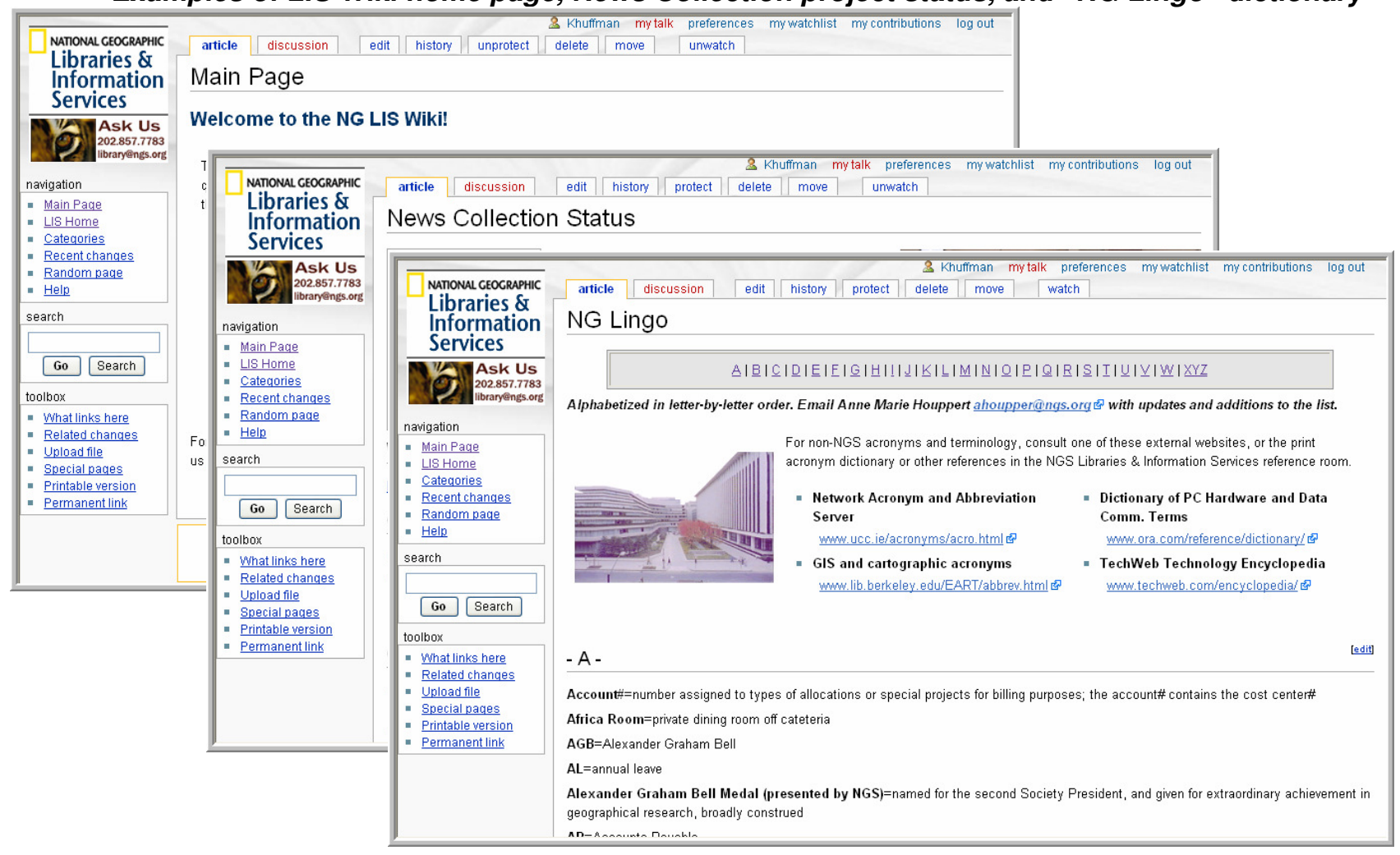


In September 2005 we started work on a Women Explorers Initiative to support content and product development for all areas of the National Geographic. We built a viable and collaborative research tool using a wiki that a cross-divisional team was invited to use to research and write about selected women. The "Women Explorers Wiki" is a research portal for internal staff which currently has nearly 250 entries and 20 contributors. We have recruited academic interns from the local library science programs to assist with research and writing entries. Members of the team officially launched the "Women Explorers Wiki" in March, 2006, during Women's History month.

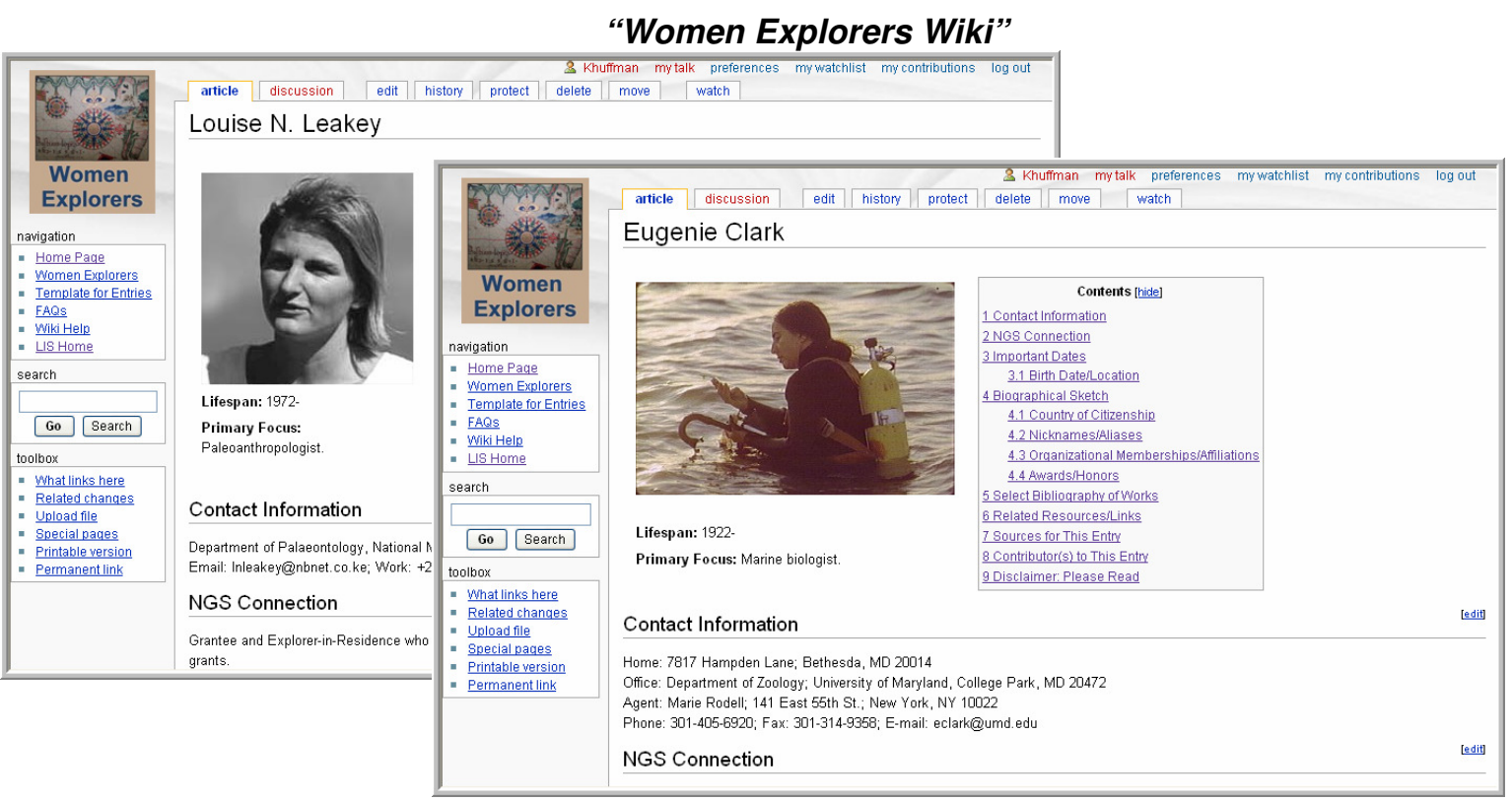

Our wikis continue to grow.... In July 2006 we launched two additional wikis: 1) a story development wiki for one of our National Geographic editorial groups to organize research material and collaborate on related ideas; and 2) a procedures manual, enabling our International Channels group the ability to easily share and maintain best practices guidelines and contact information with external producers.

Implementation of wikis work well in organizational cultures where there is a high level of trust. The organic nature of wikis is both its strength and weakness. By allowing anyone to add or edit anything there is a risk that this can lead to a chaotic information space. At National Geographic, we require contributors to create a username/login before they can contribute. We assign wiki managers and editors who develop norms and conventions for naming pages and adding content to help keep our wikis consistent.

Beyond Open Source: If Open Source applications are not supported by the organization/institution, then find ways to integrate users into supported applications. We have a number of applications that permit contributions from our user community including "NG Insider," our corporate intranet built by library staff in a Lotus content management product; our "Future Events Calendar," designed by library staff and programmed by NG contractors using Java and a SQL database; and our Trends Analysis Group's database, built by an IS\&T staff member using FileMaker Pro. We have incorporated simple templates and Web-based forms to allow for collaboration within these resources.

\section{Basic Steps for Getting Started with Examples for Academic Librarians}

This article illustrated ways Web 2.0 applications have been applied within a special library. All of the examples could easily be extrapolated to any library setting irregardless of type (i.e., special, academic, or public). Meredith Farkas, a distance librarian from Norwich University(Northfield, Vermont) developed a best practices wiki for librarians because, she said, "No one should have to reinvent the wheel when 
another librarian has already done what they're trying to accomplish" (Ishizuka, 2005). So, listed below are a few final ideas for learning more about Web 2.0, including practical suggestions for getting started:

1) Read and review recommendations and best practices from books, news sources, magazines, professional journals and/or online subscription news and research services.

2) Review Web resources. For example,

- The Earl Gregg Swem Library at the College of William and Mary in Williamsburg, Virginia, built a page of streaming RSS feeds from their blogs.

- Baylor University in Waco, Texas, has started "circulating iPods loaded with course reserves for music classes.... [Imagine how an] art library might circulate an iPod Photo with digitized images to support an art history course" (Stephens, 2005).

- Many colleges and universities including Stanford University (Palo Alto, California), Drexel University (Philadelphia) and Duke University are taking advantage of iTunes U's offerings. (Read, 2005; Stephens, 2005; Udell, 2006)

- Three useful resources full of ideas and practical applications include Meredith Farkas' Library Success Wiki: A Best Practice Wiki for librarians, a digital book from Wiki Science on starting a wiki, and Wikilndex, a wiki about wikis.

3) Take advantage of local learning opportunities and conferences through professional associations and networks.

4) Brainstorm ideas with colleagues. Initial implementation efforts do not need to be revolutionary. If the organization is interested in applying Web 2.0 ideas, then start by building guides to useful RSS feeds, podcasts, blogs, and wikis. Next, offer training and online tutorials that define the terms and enable users to gain comfort with the new ideas and tools introduced.

At the University of Maryland's College of Information Studies (clis.umd.edu), the master's students use WebCT for online course studies. WebCT is an online course management system now owned by Blackboard. A wiki could easily be developed for distance learning or enhancements to in-class work and/or project collaboration and team experiences.

5) Evaluate what resources are in-house and what the organizational culture will permit. Depending on technical expertise and staff size, a library may prefer to focus on content and services rather than on application maintenance and development. If so, then subscribe to services that set up and manage the applications. For example, the following offer hosted solutions for minimal costs: Typepad.com is a blogging service; LiveJournal.com builds communities; and JotSpot,com hosts wikis.

\section{Balancing risk and organizational culture}

In the short time National Geographic's Libraries has explored the Web 2.0 landscape, we have learned some valuable lessons:

- Make technology work to benefit users; don't make users slaves to technology.

- Define the "spaces" - what to use, when, and for what.

- Pilot ideas to show concepts and how to use the applications.

- Track metrics to measure impact.

- Deliver on promises - stay focused.

To be successful in building new tools and resources, risk-taking is key. A library must decide what their organizational culture will permit. Look for what might lend itself best to Web 2.0 applications or brainstorm new products and services. Be creative, have fun, and go for it! 


\section{References}

ABC News, Disney Online and ESPN.com. (2005, June 29). Providing extensive line-up of podcast content via the new iTunes Podcast Directory Business Wire. Retrieved July 27, 2006, from LexisNexis database.

BBCNews.com. Retrieved July 28, 2006 from http://www.bbcnews.com.

Bowers, A. (2005, Dec. 30). The year of the podcast. Business Wire. Retrieved July 27, 2006, from LexisNexis database.

Chawner, B., \& Lewis, P. H. (2006, March). WikiWikiWebs: New ways to communicate in a web environment. Information Technology and Libraries, 25(1), 33-43.

Clyde, L. A. (2005, April). Wikis. Teacher Librarian, 32(4), 54-57.

Cohen, S. M. (2006, April). The next generation of reading lists. Information Today, 23(4), 20.

Duke digital initiative: iPods at Duke. Retrieved July 28, 2006, from http://www.duke.edu/ddi/projects/ ipod.html.

Earl Gregg Swem Library at the College of William and Mary XML News Aggregator (2006, July). Retrieved July 28, 2006, from http://swem.wm.edu/resources/blogs/.

Fichter, D. (2006). Always Fresh, Keeping Your Web Site Current With News Feeds. Information Outlook, 10(4), 27-29.

Fichter, D. (2006). Using wikis to support online collaboration in libraries. Information Outlook, 10(1), 3031.

Finkelstein, E. (2005). Syndicating web sites with RSS feeds for dummies. Indianapolis, IN: Wiley.

Gibbs, M. (2006). Podzinger indexes and finds podcast content; Podzinger uses speech recognition to index podcasts for search purposes. Networld World. Retrieved July 27, 2006, from LexisNexis database.

Gomes, L. (2004, October 4). How the Next Big Thing In Technology Morphed Into a Really Big Thing. The Wall Street Journal, p. B.1.

Gordon-Murnane, L. (2005). Saying "I do" to podcasting. Searcher, 13(6), 44-51.

Huffman, K., \& Ferry, B. (2006) Web 2.0 - Making use of RSS and collaborative applications. Presentation for the Special Libraries Association Annual Conference, Baltimore, June 12-14.

Ishizuka, K. (2005). Library success a best practices. School Library Journal, 51(10), 26.

iTunes U. Retrieved July 28, 2006 from http://www.itunesu.com.

Laue, C. (2005, Sept. 18). Podcasting power. The Omaha World-Herald, 01E. Retrieved July 27, 2006, from LexisNexis database.

Library success: A best practices wiki. Retrieved July 28, 2006, from http://www.libsuccess.org.

Notess, G. (2006). The terrible twos: Web 2.0, Library 2.0, and More. Online, 30(3), 40-42.

Pew Internet and American Life Project (2005, February). iPods and MP3 Players storm the market. Retrieved July 28, 2006, from http://www.pewinternet.org/PPF/p/1047/pipcomments.asp.

Pogue, D. (2005, July 28). In one stroke, podcasting hits mainstream. The New York Times, C.1.

Read, B. (2005, July 1). Duke U. assesses iPod experiment and finds it worked - in some courses. The Chronicle of Higher Education, 51(43), A.28.

Rosenzweig, R. (2006). Can history be open source? Wikipedia and the future of the past. The Journal of American History, 93(1), 117-146.

Schiff, S. (2006). Know it all. The New Yorker, 82(23), 36-43.

Stephens, M. (2005, Spring). The iPod experiments. Library Journal Net Connect, 130, 22-24.

Udell, J. (2006). An open source sandbox. InfoWorld, 28(22), 38.

Wiki Science: How to Start a Wiki. Retrieved July 28, 2006, from http://en.wikibooks.org/wiki/ Wiki_Science:How_to_start_a_Wiki.

Wikilndex - A wiki of wikis. Retrieved July 28, 2006, from http://wikiindex.com/.

Wikipedia. Retrieved July 28, 2006, from http://en.wikipedia.org.

Young, K. (2005). Management Week - Comment - Could you be a podcast star?; Podcasting offers firms a new wy to talk to customers, but will they. VNU Business Publications Ltd., 45. Retrieved July 27, 2006, from LexisNexis database.

Karen Huffman is the Manager of Knowledge Initiatives, Libraries \& Information Services at National Geographic Society. Email: khuffman@ngs.org 